\title{
XII. Atomic structure and the spectrum of helium
}

\author{
J.W. Nicholson M.A. D.Sc.
}

To cite this article: J.W. Nicholson M.A. D.Sc. (1914) XII. Atomic structure and the spectrum of helium , Philosophical Magazine Series 6, 28:163, 90-103

To link to this article: http://dx.doi.org/10.1080/14786440708635187

册 Published online: 08 Apr 2009.

Submit your article to this journal 준

Џll Article views: 4

Q View related articles $\square$

4 Citing articles: 3 View citing articles $\square$ 
Table VII. gives the results of some of these experiments. The values of $k_{2}$ are in good agreement with the numbers obtained from Watson's experiments, but $k_{1}$ is somewhat larger.

\section{TABLE VII.}

Values of $k_{1}$ and $k_{2}$ for air at atmospheric pressures deduced from Schaffer's experiments, $b$ being the radius of the cylinder and $a$ the radius of the wire in centimetres.

\begin{tabular}{|c|c|c|c|c|c|c|}
\hline $\mathrm{V}_{0 .}$ & $\mathrm{V}$. & $b$. & $a$. & $i$. & $k_{2 \cdot}$ & $k_{1 \cdot}$ \\
\hline $8 \cdot 85$ & $14 \cdot 2$ & 385 & .00127 & 935 & 410 & \\
\hline $15 \cdot 6$ & $22 \cdot 6$ & 3.85 & .00385 & 1870 & 411 & \\
\hline $24 \cdot 5$ & $34 \cdot 2$ & $5 \cdot 85$ & .0099 & 1870 & $\ldots$ & 442 \\
\hline $57 \cdot 5$ & $66 \cdot 8$ & $5 \cdot 85$ & .052 & 3740 & $\ldots$ & 425 \\
\hline
\end{tabular}

XII. Atomic Structure and the Spectrum of Helium. By J. W. Nicholson, M.A., D.Sc., Professor of Mathematics in the University of London*.

A $\mathrm{N}$ earlier papert has indicated that the crucial test of A Bohr's theory of spectra is to be found in its application to the ordinary spectrum of helium. For the theory gives a precise specification of the constituents of a helium atom,-a specification which is in accord with the results obtained by a combination of the atomic number hypothesis of Van den Broek and the experiments of Moseley, and with those to which Rutherford and others have been led by some other lines of study. The necessity for this accordance, in fact, dictated the particular model which Bohr has used. His corresponding models for lithium and the heavier elaments have been shown to be at fault, in that they involve distributions of electrons which cannot exist by virtue of the assumptions on which the theory is founded, in spite of the fact that these assumptions are of a very general character. The success of the theory therefore rests on its application to the spectra of hydrogen and helium,-leaving out of account, for the moment, the controversial question of its application to X-ray spectra, which was partially dealt with in the earlier paper, - in so far as that application has been made. The theory must therefore stand or fall according to

* Communicated by the Author.

+ Phil. Mag. April 1914. 
its capacity to take account more completely of the spectra of these two elements, to which the analysis of the preceding paper does not apply.

The idea that series spectra are only an illustration of Planck's law, by the energy which is radiated during the passage of an atom from one state to another, is. at first sight, very promising, for it takes account of the fact that all frequencies in series spectra are differences of two functions of whole numbers. Leaving aside, in order to avoid complication, the existence of double and triple lines, satellites, and such series as those known by the name of Bergmann, we may say that for all ordinary elements whose spectra can be put into series of Rydberg's approximate type, there are three important series to be explained,-the Diffuse, Sharp, and Principal series, whose mulual relations are exhibited by the following formulæ for their frequencies, where B is Rydberg's constant,

$$
\begin{aligned}
& \text { Diffuse, } \quad \nu=\mathrm{B}\left\{\frac{1}{(1+s)^{2}}-\frac{1}{(m+d)^{2}}\right\}, \\
& \text { Sharp, } \quad \nu=\mathrm{B}\left\{\frac{1}{(1+s)^{2}}-\frac{1}{(m+p)^{2}}\right\}, \\
& \text { Principal, } \nu=\mathrm{B}\left\{\frac{1}{(1+p)^{2}}-\frac{1}{(m+s)^{2}}\right\},
\end{aligned}
$$

where $(p, s, d)$ are definite constants for the elements.

It is apparent that these series alone require, on Bohr's theory, the existence of three different types of stationary states, to which the constants $m, s, p$ are individually peculiar, and that the spectral series are formed during the passage of the atom not between different states or configurations of the same type, but between different types of states. If a helium atom only contains two electrons and a comparatively very inert nucleus, difficulties are at once apparent, for two electrons have not the necessary amount of freedom, in presence of each other, to take up three different types of relative configurations. Moreover, the spectrum of helium contains six such series, with six apparently independent constants. The atom of helium, not only according to the theory of Bohr, but in actual experiments such as those of Sir .J. J. Thomson, will not take up an extra electron, so that the neutral atom and the atom which only retains one electron are alone left to explain the entire spectrum.

It was shown in a recent paper*, that on the present form

* Monthly Nutices of R. A. S. March 1014. 
of Bohr's theory, the spectrum of helium as ordinarily manifested cannot be obtained. Moreover, it was proved that none of the possible steady states of the two electrons leads to a formula approaching that of Rydberg. The most important steady states give formulæ like Balmer's which do not preserve the Rydberg constant B. The reason for the latter property is that Bohr's deduction of the universality of $B$ in all spectra depends on the assumption that steady states exist in which one electron is at a great distance from the others, whose joint effect, with that of the nucleus, is approximately equivalent to that of a hydrogen nucleus. The paper in the Philosophical Magazine shows that this is at least impossible for three electrons in all, and therefore for lithium, which, as a matter of experience, does retain the Rydberg constant in its spectrum. The paper in the "Monthly Notices' shows that it is equally impossible for helium. Stationary states, derivable from the ordinary electrostatic forces, therefore are very limited, and if Bohr's theory is to proceed further, some change must be made.

When we consider the essentials of the theory, the limits of possible change become apparent at once. The derivation of the hydrogen formula requires that the angular momentum in the atom should be a multiple of $\frac{h}{2 \pi}$ when there is only one electron present. The same supposition is involved in obtaining the Pickering series of lines, where again there is only one electron concerned. This mulitiple property may, however, change when there is more than one electron.

The second necessity which is vital for the bydrogen formula is that the law of attraction of an electron to the nucleus is that of the inverse square. This can easily be seen by trying the law $r^{n}$. We do not get even the form of Balmer's formula unless $n=-2$. As Bohr's theory has been shown to be quite unsuccessful when there is more than one electron, we must combine the following hypotheses in any attempt to develop it further:-

(1) Nuclei attract bound electrons according to the inverse square law.

(2) Bound electrons do not repel each other according to this law.

(3) The angular momentum of an electron may cease to be $\frac{\tau h}{2 \pi}$, where $\tau$ is an integer, if there are other electrons present.

As we shall see, (2) and (3) are not alternatives, but are 
both necessary for further progress. The first hypothesis is required even for the Balmer series.

\section{The Lithium Atom.}

The conclusion of the preceding paper, that it is not possible for three electrons and a nucleus to form a lithium atom with a unit valency, after the manner of Bohr's model, was arrived at without the second hypotbesis. The electrons were regarded as repelling each other under the law of inverse square. We may now consider the possible laws of force between electrons which can admit such a configuration, in which two electrons form an inner ring, with one outside, while each electron has a constant angular momentum. We shall not require to consider whether the angular momenta of the electrons are equal, or what may be the relation between their angular velocities.

Let $f(r)$ be the law of force between two bound electrons at distance $r$ apart, and consider the three electrons at the instant when they are at the corners of a triangle ABC. Since the nucleus $O$ is very heavy, they are practically describing orbits about it, whose radii at the instant may bo called $\left(r_{1}, r_{2}, r_{3}\right)$.

If $\mathrm{AO}, \mathrm{BO}$ meet the opposite sides in $\mathrm{L}$ and $\mathrm{M}$, we can at

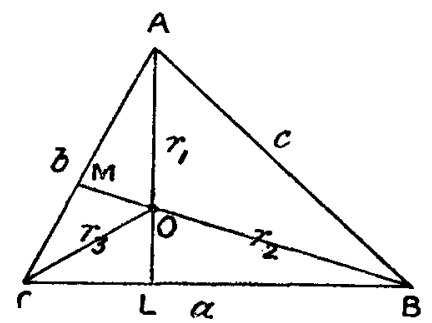

once find CL, CM. For the forces on the electron $A$ are $f(b)$ and $f(c)$ in the lines $\mathrm{CA}, \mathrm{BA}$, due to the other electrons, and the force along OA from the nucleus. The angular momentum of A does not change if the components of $f(b)$ and $f(c)$ perpendicular to $A O$ counterbalance, or

$$
\frac{f(b)}{f(c)}=\frac{\sin \mathrm{BAO}}{\sin \mathrm{CAO}}=\frac{\mathrm{BL}}{\mathrm{LC}} \cdot \frac{b}{c} \text {. }
$$

Thus

$$
\mathrm{BL}: \mathrm{LC}:: c f(b): b f(c) \text {, }
$$

and

$$
\mathrm{CL}=\frac{a b f(c)}{b f(c)+c f(b)} \text {. }
$$

Similarly

$$
\mathrm{CM}=\frac{a b f(c)}{c j(a)+a f(c)}
$$


If the line CB is taken as an axis of $x$ in a Cartesian system with $\mathrm{C}$ as origin, the equations of $\mathrm{AL}, \mathrm{BM}$ become

$$
\begin{aligned}
& y=\frac{b \sin \mathrm{C}(x-\mathrm{CL})}{b \cos \mathrm{C}-\mathrm{CL}}, \\
& y=\frac{\mathrm{CM} \sin \mathrm{C}(x-a)}{\mathrm{CM} \cos \mathrm{C}-a},
\end{aligned}
$$

and the coordinate $x$ of the point $O$ becomes on solntion,

$$
x=\frac{a \mathrm{CL} \cdot(\mathrm{CM}-b)-b \cdot \mathrm{CM} \cos \mathrm{C}(a-\mathrm{CL})}{\mathrm{CL} \cdot \mathrm{CM}-\bar{a} b} .
$$

If $r_{2}=r_{3}$, so that two of the electrons are in a ring, $x$ is $\frac{1}{2} \alpha$, and this must be true approximately if the deviations of the two electrons from their ring exist, but are not large.

'This condition reduces to

$$
a^{3} b+a^{2} \mathrm{CL} . \mathrm{CM}-2 a^{2} b . \mathrm{CL}=\mathrm{CM}(a-\mathrm{CL})\left(b^{2}+a^{2}-c^{2}\right) ;
$$

and substituting for CL and CM, the law of force $f(r)$ must be consistent with

$$
\begin{gathered}
a\{b f(c)+c f(b)\}\{c f(a)+a f(c)\}=a^{2} b f^{2}(c)+2 a b c f(a) f(c) \\
+c\left(b^{2}+a^{2}-c^{2}\right) f(b) f(c)
\end{gathered}
$$

for all values of $a, b$, and $c$. No law which is a power of $r$ will ordinarily serve, for writing $f(r)=\lambda r^{n}$, we have

$$
\left.a\left(b c^{n}+c b^{n}\right)\left(c a^{n}+a c^{n}\right)=a^{2} b c^{2 n}+2 a b c . a^{n} c^{n}+c b^{2}+a^{2}-c^{2}\right) b^{n} c^{n}
$$

or on reduction,

$$
\left(b^{2}-c^{2}\right) c^{n-1} b^{n-1}=a^{n+1}\left(b^{n-1}-c^{n-1}\right) .
$$

The case $b=c$ is a solution for any such law. If $b$ is not equal to $c$, any case of $n$ negative (and the case $n=0$ ) makes one side of the equation essentially positive and the other essentially negative, so that it cannot be satisfied. The case $n=1$ compels $b$ and $e$ to be equal. $n=2$ gives $a=[b c(b+c)]^{\frac{1}{3}}$, unless $b=c$, and in fact, laws of direct distance beyond the first are formally possible. But they cannot be permissible from physical considerations, for the repulsion between electrons cannot be thought of as increasing with their distance apart, when that distance is comparable with the atomic radius, as in Bohr's rings of electrons.

If the distance apart were comparable with the radins of an electron, of order $10^{-13}$, such a possibility might be admitted, and in a subsequent paper it will be found to lead to valuable conclusions. But here we are not concerned with possible nuclear structure, of which this is a question, but 
with rings of more ordinary atomic size, for which the only conclusion is that $b=c$. This signifies that the electron $A$ is always equidistant from the other two which form a ring. It therefore rotates with the same angular velocity, and from this point the reader can easily supply the proof that all three electrons must be in one ring. The conclusion extends not only to the law $\lambda r^{n}$ for the force between two electrons, but to any law $f(r)$ which can be expressed in a power series, - such a law, for example, as a mixed inverse square and cube, of the form

$$
\frac{e^{2}}{r^{2}}+\frac{\lambda_{1}}{r^{3}}+\ldots
$$

We must finally suppose that the law of force between electrons bound in an atom which can admit Bohr's lithium model is either-

(1) No force at all, which evidently constitutes a solution, or

(2) Any law whatever, provided that the three electrons are all in one ring.

This last alternative cannot take account of the valency of lithium, as in the last paper, whose investigation can be extended.

But, in addition, direct distance laws are formally possible for electrons which are actually on the confines of the nucleus. These, however, are not rings of electrons in the sense required by Bohr's theory, but are $\beta$-particles. They would be too close to the nucleus to be capable of behaving with it otherwise than as neutral doublets. We do not need to consider their possibility any further at present. For we cannot effect a compromise, snch as would be suggested by putting two electrons close to the nucleus (within a distance $10^{-13}$ ) under this law, and the third as an outer valency electron of lithium at a distance $10^{-8}$, acted on by the other two according to the inverse square law. For such an arrangement would give lithium, on Bohr's theory, a spectrum which could not be distinguished from that of bydrogen. Coplanar rings in Bohr's sense are therefore only possible if bound electrons exert no force on each other,-for the case of lithium at least. $W e$ can generalize this result from lithium to any other element, but it is not necessary to give the details of the argument. If the possibility of zero force is admitted, there is no limit to the number of such rings which are possible, or to the number of electrons which each can contain. The atom becomes remarkably indefinite, for any electron is only acted upon by the nucleus, and its 
behaviour is as simple as that of the electron in a hydrogen atom. The consequences of such an hypothesis in connexion with spectra will appear later. At present it has only been shown that it is the only hypothesis which will, on Bohr's theory, allow a coplanar-ring structure of the ordinary elements.

We may notice that this hypothesis is a step towards Sir J. J. 'Thomson's conception of tubes of force in the atom. The relation between the two points of view is as follows. If an electron when free has tubes of force, with some material significance, radiating from it, it may when bound have these tubes diverted to pass into the nucleus, and all the electrons in an atom being in this connexion with the nucleus, cannot exert force on each other. An accelerated electron might radiate if its tubes extended to a distance, but not when they all passed to the nucleus. This connexion between two points of view is interesting, but may have no special significance.

The Spectrum of Helium.

On the supposition that a helium atom, when neutral, contains only two electrons and a nucleus $2 e$, and that the spectrum of the atom when one electron has disappeared is that calculated by Bohr, and previously believed to be due to hydrogen, we are compelled to suppose that the more normal helium spectrum is produced by a neutral atom. For a helium atom, on Bohr's theory and according to Sir J. J. Thomson's experiments, will not take up a third electron. The neutral atom must therefore possess six types of stationary states, as there are six types of function to which the application of the combination principle gives the helium spectrum. We shall attempt to arrive at any one of these functions by Bohr's method in the most general manner which is yet consistent with the theory of the spectra of the hydrogen atom and the positively charged helium atom. The best series is evidently the sharp or second subordinate series of parhelium, given by the very accurate Hicks' formula

$$
n=27174 \cdot 917-109726 \cdot 0 /\left\{m+\cdot 861181-\frac{\cdot 007809}{m}\right\}^{2} \text {, }
$$

where $n$ is the wave number. For the Rydberg constant is in this case $109726^{\circ}$, which is almost precisely Bohr's estimate 109725 instead of the usual 109675 of hydrogen. If the theory is correct, 109725 is the theoretical value for elements heavier than hydrogen. We deduce that one of the types of stationary states concerned in the production 
of this series is formed by the radiation, during the collection of the atom from infinite dispersion, of an amount of energy

$$
\mathrm{W}=\frac{2 \pi^{2} m e}{h^{2}} /\left(m+\cdot 861181-\frac{\cdot 007809}{m}\right)^{2} .
$$

Now if there is any force whatever between the two electrons, they can only have stationary states when they are continually in a line with the nucleus, provided that their orbits are in the same plane. This can be proved iminediately, on the supposition that they move with constant angular momenta. When they are in this line with the nucleus continually, their angular velocities must be equal;

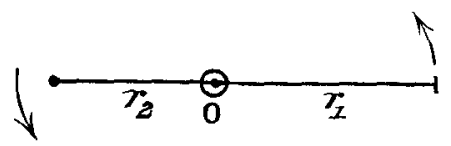

and since their angular momenta are specified, the radii must be in a constant ratio, which is one of equality if the angular momenta are equal. Let the rapulsion between them, when at distance $r$ apart, be $\frac{\lambda}{r^{n}}$. Then their orbits are given by

$$
\begin{array}{ll}
\frac{d^{2} u_{1}}{d \theta^{2}}+u_{1}=\left\{\frac{\nu}{r_{1}^{2}}-\frac{\lambda}{\left(r_{1}+r_{2}\right)^{n}}\right\} \frac{1}{h_{1}^{2} u_{1}^{2}}, & u_{1}=\frac{1}{r_{1}}, \\
\frac{d^{2} u_{2}}{d \theta^{2}}+u_{2}=\left\{\frac{\nu}{r_{2}^{2}}-\frac{\lambda}{\left(r_{1}+r_{2}\right)^{n}}\right\} \frac{1}{h_{2}^{2} u_{2}^{2}}, & u_{2}=\frac{1}{r_{2}} .
\end{array}
$$

In the 'Monthly Notices"* it is proved that these orbits do not exist unless they are identical, under the inverse square law. For other laws, we may proceed as follows. Let the angular momenta $m h_{1}$ and $m h_{2}$ be given by $h_{1}=\alpha^{2} h, h_{2}=\beta^{2} h$, $\alpha^{2}$ and $\beta^{2}$ being in a ratio of integers on Bohr's view, but more generally, perhaps, in other constant ratios. Then if $\omega$ is the angular velocity,

and

$$
r_{1}^{2} \omega=\alpha^{2} h, \quad r_{2}^{2} \omega=\beta^{2} h ;
$$

$$
r_{2}=r_{1} \frac{\beta}{\alpha}, \quad u_{2}=u_{1} \frac{\alpha}{\beta}
$$

* March 1914.

Phil. Mag. S. 6. Vol. 28. No. 163. July 1914. 
so that

$$
\begin{gathered}
\frac{d^{2} u_{1}}{d \theta^{2}}+u_{1}=\left\{\nu u_{1}^{2}-\lambda u_{1}^{n} \frac{\alpha^{n}}{(\alpha+\beta)^{n}}\right\} \frac{1}{\alpha^{1} / h^{2} u_{1}^{2}}, \\
\frac{\alpha}{\beta}\left\{\frac{d^{2} u_{1}}{d \theta^{2}}+u_{1}\right\}=\left\{v \frac{\alpha^{2}}{\beta^{2}} u_{1}^{2}-\lambda u_{1}^{n} \frac{\alpha^{n}}{(\alpha+\beta)^{n}}\left\{\frac{\beta^{2}}{\beta^{4} h^{2} u_{1}^{2} \alpha^{2}}\right\} ;\right.
\end{gathered}
$$

and therefore for all values of $u_{1}$, by division,

$$
\frac{1}{\alpha^{3} \beta}\left\{\nu u_{1}^{2}-\lambda u_{1}^{n} \frac{\alpha^{n}}{(\alpha+\beta)^{n}}\right\}=\frac{1}{\alpha^{2} \beta^{2}}\left\{v \frac{\alpha^{2}}{\beta^{2}} u_{1}^{2}-\lambda u_{1}^{n} \frac{\alpha^{n}}{(\alpha+\beta)^{n}}\right\} \text {. }
$$

Excluding $n=2$, already dealt with in the other paper *, this compels $\alpha$ to he equal to $\beta$, so that the angular momenta are equal. Then $r_{1}=r_{2}$, and the electrons are continually equidistant from the nucleus.

For orbits in one plane, therefore, under any law of electronic repulsion, the electrons are equidistant from the nucleus; and thus they move in the same circular or elliptic path, or keep pace witb each other in two equal paths with the same major axis, as in the diagram given in the Phil Mag. paper $\dagger$.

By analysis already given, and capable of extension $\ddagger$, the same conclusion can be inferred for non-coplanar paths, of which the figure gives an illustration, showing such possible paths of each electron and of the nucleus round the axis of

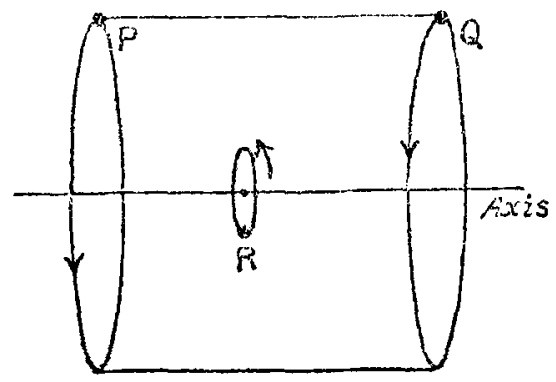

the atom. It is evident that it applies to any law of force whatever which can be expressed in a power series. The details of the analysis can ersily be filled in by the reader. We conclude, finally, that for any law of force between electruns, the two in a helium atom must be equidistant from the nucleus throughout any of Bohr's steady states.

* Phil. Mag. April 1914.

t'Monthly Notices,' March 1914. 
Bohr has noted * the difficulty of any other supposition in the case of two electrons, although apparently he has not considered the example in the figure. A more generai one can be obtained by rotating the whole atom about an axis perpendicular to the axis of the atom, but as stated in the 'Monthly Notices'†, even this set of configurations does not give anything resembling the helium spectrum when the law of force between the electrons is the inverse square. In order, therefore, to explain the helium spectrum, this law must be abandoned.

Let us now follow out the consequences of the law of the inverse $n$th power, as a preliminary to any other law which can be imagined. We may suppose the path of the electrons to be circular, as under the inverse square law, wilhout a real loss of generalicy. Taking the case in which the orbits are coplanar, we see from the above reasoning that the electrons are equidistant from the nucleus, and their circular orbits are therefore identical. If the force between the electrons is $\frac{\lambda}{r^{n}}$ where $r$ is the distance apart, the angular velocity and radius

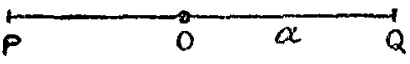

are given by

$$
\begin{aligned}
& m a \omega^{2}=\frac{2 \nu^{2}}{a^{2}}-\frac{\lambda}{(2 a)^{n}}, \\
& m a^{2} \omega=\frac{7 h}{2 \pi},
\end{aligned}
$$

where the second is the condition of angular momentum. The sum of the kinetic and potential energies is, if $n$ is not unity,

$$
\mathrm{C}+m a^{2} \omega^{2}-\frac{4 e^{2}}{a}+\frac{\lambda}{n-1(2 a)^{n-1}},
$$

where $\mathrm{C}$ is the energy in a state of infinite dispersion. This becomes

$$
\mathrm{C}-\frac{2 e^{2}}{a}-\frac{(n-3) \lambda}{(n-1) 2^{2} a^{n-1}}
$$

The energy radiated in forming the atom is

where $n$ is given by

$$
W=\frac{2 e^{2}}{a}+\frac{(n-3) \lambda}{(n-1) 2^{n} a^{n-1}},
$$

$$
\frac{\tau^{2} h^{2}}{4 \pi^{2} m}=a\left(2 \epsilon^{2}-\frac{\lambda}{2^{n} a^{n-2}}\right)
$$

- Phil. Magr. July 1913. 
If $n$ is not $2, \lambda$ contains the $(n-2)$ th power of some constant length, and it is very difficult to imagine what the length could represent physically. For it is of the same order as the diameter of the atom. This alone is perhaps sufficient to remove the possibility of any law except the inverse square. Nevertheless, we may give a brief account of some other laws. For the inverse cube, $n=3$, and

$$
\begin{aligned}
\frac{\tau^{2} h^{2}}{4 \pi^{2} m} & =2 a e^{2}-\frac{\lambda}{8}, \\
W & =\frac{4 e^{4}}{\frac{\lambda}{8}+\frac{\tau^{2} h^{2}}{4 \pi^{2} m}}
\end{aligned}
$$

Spectrum lines on Bohr's theory would then show series of the type

$$
h \nu=\mathrm{A}-\frac{\mathrm{B}}{a^{2}+\beta^{2} \tau^{2}},
$$

where $\tau$ is integral. Such series are unknown. Similar conclusions follow trom the inverse fourth power. In fact, for any inverse power beyond the second, the radius is a function of $\tau^{2}$, not $\tau$ itself, and so is the variable part of any ensuing series formula. This is quite at variance with fact. Any composite inverse law, such as a mixed square and cube, produces the same result. A glance at the accurate Hicks' formula already quoted for helium, which is quite typical, will show the impossibility of a theory which only introduces the variable integer of series spectra in the forms of even powers. When $n=1$, the potential energy is

$$
-\frac{4 e^{2}}{a}+\lambda \log (2 a),
$$

and cannot be admitted as a possible value, on account of the behaviour of the logarithm.

Thus on no possible law of electronic repulsion can we derive any series for helium similar to the usual forms. The inverse square, as shown in the 'Monthly Notices,' usually leads to series like Balmer's which do not correspond with fact. We cannot indeed derive the forms

$$
\frac{N}{\left(\tau+\alpha+\frac{\beta}{\tau}\right)} \text { (Hicks) or } \frac{\mathrm{N}}{\left(\tau+\alpha+\frac{\mathrm{B}}{\tau^{2}}\right)^{2}} \text { (Ritz), }
$$

or anything analogous to them for the variable part of series spectra under the combined hypotheses that the angular 
momentum of an electron is $\frac{\tau h}{2 \pi}$ ( $\tau$ integral), and that the law of electronic repulsion has any possible form.

We must thus modify the law of angular momentum. It must be so changed that the angular momentum still contains the factor $\frac{h}{2 \pi}$, or the whole relation with Planck's theory is lost. But at the same time, it must cease to be an integral multiple of $\frac{h}{2 \pi}$. We write, therefore, $f(\tau) \frac{h}{2 \pi}$ for the angular momentum, where $f(\tau)$ is a function not only of an integer $\tau$, but also of the nuclear charge and the number of electrons in the atom.

With this specification, we can resume consideration of the inverse square law. It is easily shown that the energy radiated in forming the atom is

$$
\mathrm{W}=\frac{2 \pi^{2} m e^{4}}{h^{2}} n\left(\mathrm{~N}-\frac{1}{4} \mathrm{~S}_{n}\right)^{2}\left\{\frac{1}{f^{\prime}(\tau)}\right\}^{2},
$$

where $\mathrm{N} e$ is the nuclear eharge, and $n$ the number of electrons. We must deal first with the factor $n$. Unless $f(\tau) \propto \sqrt{n}$, the Rydberg constant cannot be preserved, provided that only one quantum of energy is evolved in passing from this state to any other. If we modify the theory, however, so that every electron emits a quantum, $\frac{W}{n}$ is the determining
feature in the frequency emitted.

An angular momentum depending on the square root of the number of electrons can hardly. be imagined as possible: so that the theory should probably be thus modified. This modification was indicated in the earlier paper* as necessary to Moseley's interpretation of his X-ray results. It is here required for ordinary spectra as well.

But the Rydberg constant is still not preserved unless $f(\tau)$ is proportional to $\mathrm{N}-\frac{1}{4} \mathrm{~S}_{n}$. This is an even more difficult connexion to imagine, for it would destroy the whole meaning of the relation of the atom to Planck's theory. Moreover, when $n=1, f(\tau)$ has all integral values and not half integral ones if Bohr's theory of the Pickering series is correct. If $f(\tau)$ were necessarily proportional to $N-\frac{1}{4} S_{n}$, it could only have values which were multiples of 2 in this case $\left(\mathbb{S}_{n}=0\right)$, and this would cut out the Pickering series. The only conclusion therefore is that we cannot preserve Rydberg's constant by any theory which ascribes the Pickering series to helium. Once it is so ascribed, the

* P. 562 . 
constant cannot be retained for any other helium atom with a different charge,-when the law of force between the electrons is the inverse square. Now the Pickering spectrum is at least due to hydrogen or helium. Bohr's theory cannot deduce it for hydrogen, and can only do so for helium by a process which destroys the possibility of even preserving Rydberg's constant, or a constant approximately equal to it, for the rest of the helium spectrum. Thus with any modification which we may make of the law of angular momentum, the inverse square law between electrons cannot be retained.

But the inverse $n$th power of the distance between the electrons is equally of no service, and is subject to all the difficulties mentioned before, which are decisive against it. There is no necessity to give the analytical treatment, which can easily be supplied by the reader. Bohr's theory cannot therefore explain the helium spectrum, or indeed any other series spectrum, by any modification which will retain the simpler theory of hydrogen and the Pickering series, when there is force between bound electrons. If, on the other hand, no such force exists, we obtain as the variable part of a series spectrum from an atom of nucleus $\mathrm{N} e$,

$$
\frac{2 \pi^{2} m e^{4}}{h^{3}} \frac{\mathrm{N}^{2}}{(f(\tau))^{2}}
$$

where $\tau$ is an integer. Every electron is independent of every other, but yet the angular momentum may depend on the number of electrons, if the nucleus has the property of compelling the existence of a definite angular momentum, or set of angular momenta, about it. In order to preserve the universal constant of series, $\mathrm{N}$ must divide into $f^{\prime}(\tau)$; but this involves either that it shall do so in helium with one electron, also thus excluding the Pickering series, or else that half integers may be used for $\tau$ in the ordinary helium series, which is quite at variance with the known spectrum of helium. We are supposing, of course, as is necessary for any theory of actual spectra, that

$$
f(\tau)=\mathrm{A}(\tau+\alpha+\psi(\tau))
$$

where A and a are constants and $\psi(\tau)$ is a small quantity. Into $\mathrm{A}$ the division of $\mathrm{N}$ is to take place. Preservation of the universal constant involves that $A=1$ before or after division, and the alternatives are therefore $A=1, A=N$, which lead to the conclusions above.

All other possible stationary states of a helium atom under. any laws of force are subject to the same analysis, and 
therefore helium does not possess a nucleus charge $2 e$, or Bohr's theory of series is incorrect. But since the whole argument can be extended to atoms with nuclei $3 e$ and $4 e$. we conclude that this theory cannot give the helium spectrum from any atom, and is incapable therefore of further development in the interpretation of spectra. The fact that this incapacity is associated with a similar one in connexion with the sequence of chemical properties of the elements is very decisive.

It might perhaps be urged that only the total angular momentum in the atom is a multiple of $\frac{h}{2 \pi}$ and not that of any individual electron, as supposed, after $\mathrm{Bohr}$, in all the preceding work. In that case the problem is one of "periodic orbits," but as such it can hardly, by its solution, lead to a definite picture of the atomic processes which can be called a theory of spectra. For the essential feature of the anguiar momentum principle is that it makes the atom definite, by only admitting a certain number of possible radii or angular velocities, whether constant values with an actual existence or mean values associated with non-circular orbits. If, as in Bohr's fundamental assumption, bound electrons do not radiate energy in their stationary states, and thus experience nodrag on their motion, the total angular momentum of the atom must remain constant. A knowledge of this constant does not go far in fixing the atomic structure when there is more than one electron. More conditions must be imposed in order to obtain a definite atom, except in special cases of the simplest periodic orbits which are possible. These are the cases investigated in this paper, in which the angular momentum is shared in a simple way between the electrons. They must be the cases of most frequent occurrence in actual atoms, and the fact that they do not show the spectra which theory requires is in itself sufficient to show that spectra will not be obtained from the less likely though more indefinite cases. No generalization of the theory on these lines can therefore bope to be successful, and we must conclude that it cannot develop in the manner which its earlier succsss appeared to foreshadow. At the same time, the connexion between the Rydberg constant and Planck's constant is so close that it is difficult to believe that it is not real. But such a reality does not contain a corresponding reality for the process by which the form of the bydrogen spectrum is derived. It can be derived, for example, in its entirety, also from the theory of Ritz. 